Computer Optics and Nanophotonics

\title{
Diffraction lens in imaging spectrometer
}

\author{
Blank V.A., Skidanov R.V. \\ Image Processing Systems Institute, Russian Academy of Sciences, \\ Samara State Aerospace University
}

\begin{abstract}
A possibility of using a diffractive lens as the simplest imaging spectrometer was considered. An experimental construction of the spectral imaging for white, red and green LEDs was conducted. When processing the images the influence of the scattered components of the initial point and nearly points of the image was taken into consideration. There was made a comparison of the received spectral distribution with the spectra of these LEDs, received by means of spectrometer. Root-mean-square error (RMSE) from 8 to $13 \%$.
\end{abstract}

Keywords: diffraction lens, imaging spectrometer, spectral image.

Citation: Blank V.A., Skidanov R.V. Diffraction lens in imaging spectrometer. Proceedings of Information Technology and Nanotechnology (ITNT-2015), CEUR Workshop Proceedings, 2015; 1490: 17-26. DOI: 10.18287/1613-00732015-1490-17-26

\section{Introduction}

In the past decade, in applied spectroscopy there have been intensively developing the new methods, which allow getting and analyzing the spectroscopic information about an object with a spatial resolution, providing the reception for each small area (point) the two-dimensional image of the object on the input aperture of the optical spectrum instrument. In scientific literature such methods are called imaging spectroscopy, three-dimensional matrix containing the dependence of intensity of light from the two spatial and spectral coordinates are called spectral images [1].

In most modern imaging spectrometers optical system forming that imaging and dispersive element are clearly separated. As a dispersive element in imaging spectrometer can be used a prism as well as diffraction grating [2,3]. However, there is a diffractive optical element that can combine these two functions. For quite a long time the attempts have been made to use a diffractive lens in various applications [49]. Including the works dedicated to the spectral properties of the diffractive lens [9]. However, high chromatic aberration does not allow the use of the diffraction lens in the imaging systems [6]. The diffraction lens builds images for different wavelengths at different distances. However, this disadvantage of the diffractive lens is more likely to be an advantage, when using the diffraction lens as a basis for the spectrometer [10-12]. In this paper [10,11] a spectrometer using a diffractive lens as a dispersive element is regarded. The light focalized by the diffractive lens passes through the 
diaphragm and falls on the photodiode, i.e. the spectrometer observed in the papers $[10,11]$ is not imaging. In the papers $[12,13]$ the spectrometer based on a combination of diffraction lens and diffraction grating is observed, due to which, a really compact construction is obtained. However, this spectrometer is not an imaging one as well. In the paper [14] there is a simple research on the possibility of using the diffractive lens as a basis for the imaging spectrometer. However, in the paper [14] the authors reviewed rather simple work situation of such spectrometer when the initial object is several multicolored characters on the screen, each of which radiates in a relatively narrow spectral range. Besides, for convenience the symbols are spatially separated which only facilitates the task. In addition, no spectrum was given. In the paper [15] was presented an extended research and was given measured spectra for the laser beams (lasers $\mathrm{He}-\mathrm{Ne}$ and $\mathrm{Cr}-\mathrm{Ne}$ ). On the imaging spectrometer a spectrum of a point object was measured, consisting of one spectral line. I.e. it is possible to draw a conclusion from the papers $[14,15]$ that the diffraction lens actually allows getting a spectral image. To confirm the efficiency of such configuration it is necessary to conduct a research on the magnitude of error starting from which is built a spectral image in the spectrometer based on the diffraction lens. In the given article the constructions errors of the spectral image of the white LED by means of the diffraction lens are examined. In addition, a spectral image for the structure of several LEDs with different spectral characteristics is built.

\section{Experimental reception of a series of images by means of diffraction lens}

To test the possibility of restoring the spectral distribution for every dot of the image for the objects with a wide spectrum there was conducted an experiment in which was built a series of white LED images by means of diffraction lens with a focal length of $50 \mathrm{~mm}$ for a wavelength of $500 \mathrm{~nm}$. For the experiment, a four-leveled diffraction lens was used, described in [6]. The lens diameter was $8 \mathrm{~mm}$.

Fig. 1 Shows the optical scheme of the experiment.

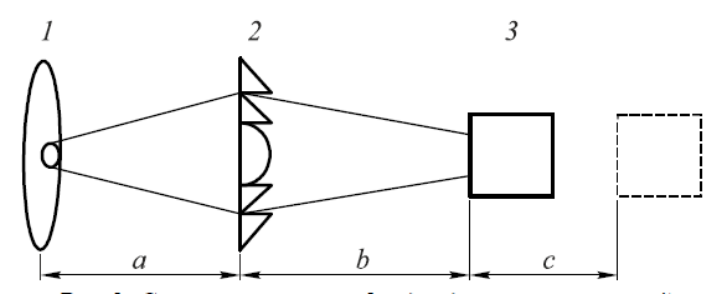

Fig. 1. - Installation diagram. 1 - diode disposed at a distance from the diffraction lens 2, 3 CCD camera disposed at a distance $b=40 \mathrm{~mm}$ from the diffraction lens. CCD camera is installed on a moving table with an approach increment of $0.5 \mathrm{~mm}, c=20 \mathrm{~mm}$

Whilst the distance from the LED to the diffraction lens $\mathrm{a}=2000 \mathrm{~mm}$ was less than the distance from the diffraction lens to a CCD camera (VIDEOSCAN-2-205) with a resolution of $1390 * 1040$. In the experiment, the camera moved from $40 \mathrm{~mm}$ to $60 \mathrm{~mm}$ with a step of $0.5 \mathrm{~mm}$. Each image corresponds to its own wavelength. For convenience, the diode was located close to the optical axis. 
Fig. 2 shows a part of white LED spectrum, obtained by the spectrometer MS7501 (fig. 2). A relative error of spectrum measurement on this spectrometer is not more than $2 \%$.

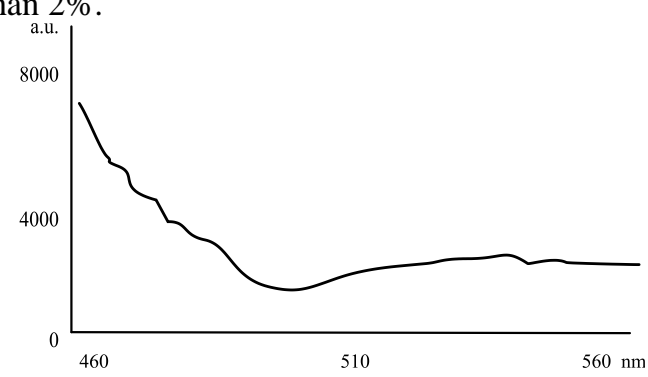

Fig. 2. - A segment of white LED spectrum (standard)

The spectral distribution in Fig. 2 hereinafter to be used as a standard spectrum for comparison of the spectra of individual image points of the LED obtained by means of diffractive lens.

Fig. 3 shows the examples of the LED images at several distances, which correspond to the wavelengths of $481 \mathrm{~nm}, 505 \mathrm{~nm}$ and $550 \mathrm{~nm}$. The image size of the LED in fig. 3 was nearly 100 micrometer (22 pixels on CCD array).

a)

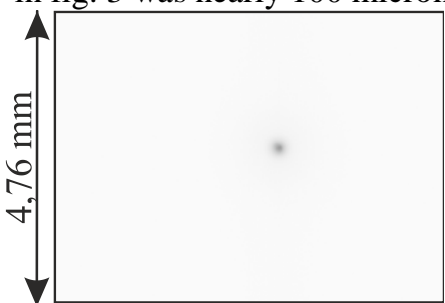

a)

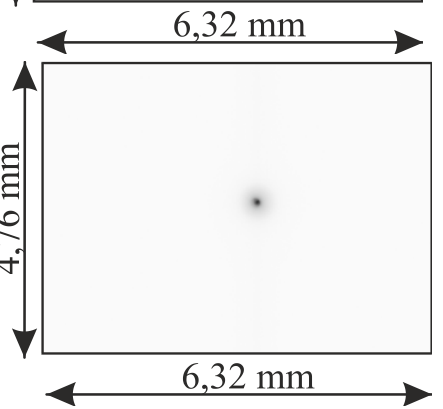

c) b)

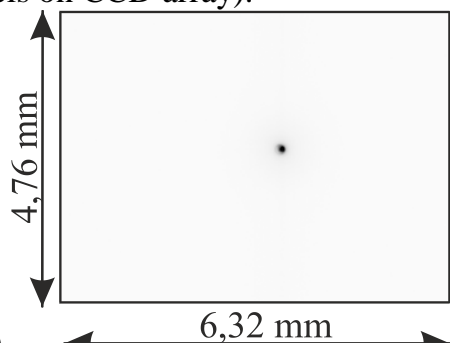

Fig. 3. - Experimental images: $481 \mathrm{~nm}(\mathrm{a}), 505 \mathrm{~nm}(\mathrm{~b}), 550 \mathrm{~nm}(\mathrm{c})$

\section{Processing of the experimental images to obtain a spectral image}

However, it is not allowed to use the experimental images directly without complementary processing. The matter is that due to the change of distance from lens to image changes the scale. I.e. the LED image shifts in relation to the center of the image pattern and additionally changes its size. It cannot be avoided even if the LED image is positioned at the exact center. In this case what is left is a change if the 
image's scale. To overcome this effect, the experimental images were subjected to geometric transformation of tension / compression relative to the center of the picture with a coefficient equal to the ratio of the distances. Fig. 4 shows the LED images at several distances corresponding to different wavelengths $(481 \mathrm{~nm}, 505 \mathrm{~nm}, 550 \mathrm{~nm})$ after the geometric transformation. After this transformation, the image of any size keeps the size and position for any wavelength.

a)

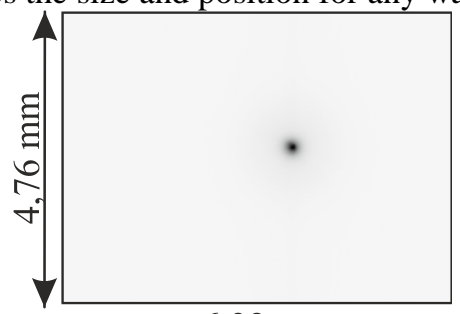

)

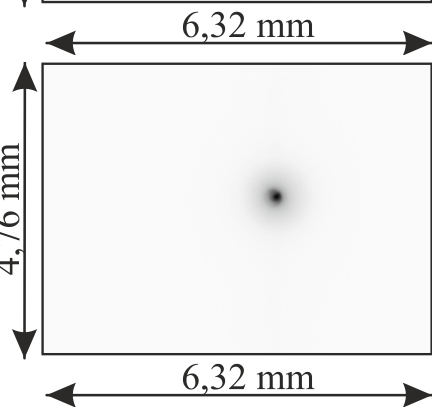

c) b)

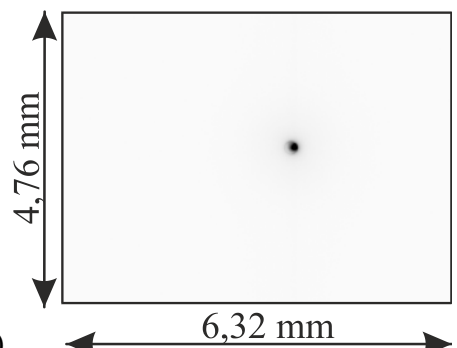

Fig. 4. - Experimental images after geometric transformation: $481 \mathrm{~nm}(\mathrm{a}), 505 \mathrm{~nm}(\mathrm{~b}), 550 \mathrm{~nm}$ (c)

For non-imaging spectrometer with a diffraction lens [10], the procedure of the spectrum recovery according to the measurement sequence is quite simple. In the paper [10] itself the authors do not make an additional processing to remove the scattered components. But having obtained the sequence of the intensity distributions at different distances $I_{j}(x, y)$ it is necessary to solve a system of linear equations.

$$
I_{j}(x, y)=\sum_{i=1}^{N} I\left(x, y, \lambda_{i}\right) \frac{A}{A+\left(b_{j}-b_{i}\right)^{2} k_{i}^{-2} \pi / 4}, j=\overline{1, N},
$$

where $I\left(x, y, \lambda_{i}\right)$ are the elements of spectral image, $b_{i}, b_{j}$ are distances corresponding to the wavelengths, $k_{i}-f$-number of the diffractive lens, $I_{j}(x, y)-$ intensity distributions taken at distances $b_{j}, A-$ square of pixel in CCD array. $F$ number for the diffractive lens is determined by the formula $k_{i}=d / b_{i}$, where $d$ is lens diameter. The formula (1) was derived from geometrical considerations. For a precise focus, when the distance $b_{j}$ corresponds to the wavelength we supposed that the size of aberration image is smaller than the size of pixel of the CCD array (4.65 micrometer*4.65 micrometer). It is actually so, the lens diameter is $8 \mathrm{~mm}$ at a focal length of $50 \mathrm{~mm}$, i.e. the size of the diffraction aberration image will be about 4 
micrometers. At displacement of over a certain distance, we calculated the size of the spot based on the geometrical considerations. For the imaging spectrometer, this system of equations becomes more complicated because it is necessary to take into account the contribution of neighboring dots to the diffuse component on the object

$$
I_{j}(x, y)=\sum_{i=1}^{N} \int_{u^{2}+v^{2}<R^{2}} \frac{I\left(x+u, y+v, \lambda_{i}\right) A}{A+\left(b_{j}-b_{i}\right)^{2} k_{i}^{-2} \pi / 4} d u d v, j=\overline{1, N} \text {, }
$$

where $R=\left(b_{j}-b_{i}\right) k^{-1}$ is the blur size of a dot at focus displacement, when $\mathrm{j}=\mathrm{i}$, in the calculations we shall assume that aberration image coincides in radius with pixel. In the discrete case, the integral in formula (2) degenerates into a sum over the aberration image's dot for the appropriate wavelength. The system of equations (2) for a wide spectral range has a high computational complexity. The number of dots in a discrete variant according to which the summation is performed can reach several thousands.

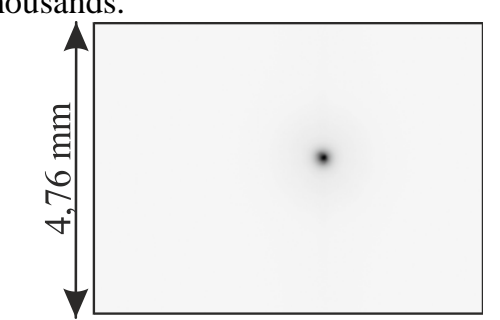

a)

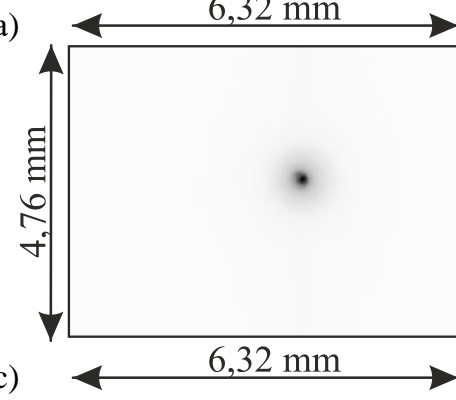

b)

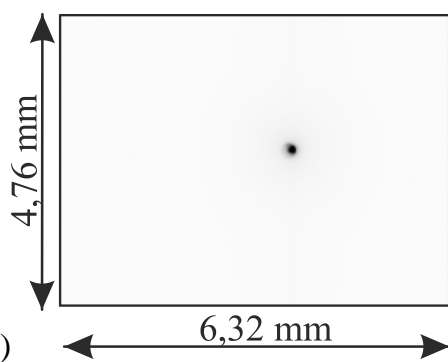

Fig. 5. - Processed images: $481 \mathrm{~nm}(\mathrm{a}), 505 \mathrm{~nm}(\mathrm{~b}), 550 \mathrm{~nm}$ (c)

For the case where the object for which the spectral image is formed is relatively compact, the computational complexity reduces. Thus, for the LED (fig. 3) it is necessary to take into account just a few tens of dots. Besides, for the spectrum recovery process there was considered a different diffractive effectiveness of the diffractive lens for different wavelengths, which is calculated according to the formula [15]

$$
\eta=\frac{\sin ^{2}\left[(n-1) h \frac{\pi}{\lambda}-\pi\right]}{\left((n-1) h \frac{\pi}{\lambda}-\pi\right)^{2}} \frac{\sin ^{2}\left[(n-1) h \frac{\pi}{4 \lambda}\right]}{\left((n-1) h \frac{\pi}{4 \lambda}\right)^{2}},
$$


where $\eta$ is spectral sensitivity, $h$ is relief depth, $n$ is a refractive index of the lens material.

The spectral sensitivity of CCD array [16] was also taken into consideration. Thus, fig. 5 shows the processed images.

As a result of the solution of equations (2), the following spectral distribution was obtained for one of the dots for the diode image (fig. 6).

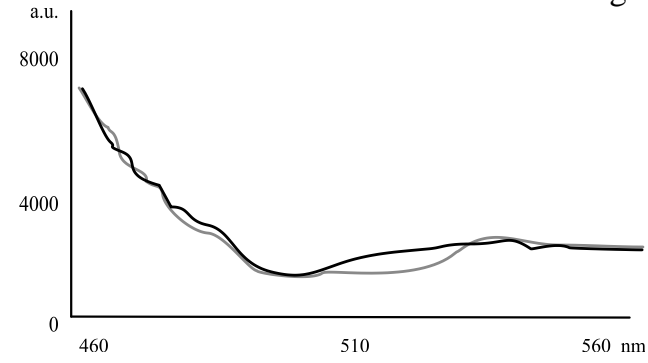

Fig. 6. - Diode spectrum obtained by means of spectrometer and by the results of processing a set of experimental images according to the formula (2)

RMSE of recovery range was $11 \%$. However, for such compact objects as LED it is possible to use a simpler means of obtaining a spectral image with an insignificant loss of accuracy. After obtaining a geometrically correct image (fig. 4), a scattered component can be removed from all images. To do that, there was determined an average number of the intensity of the scattered light outside the LED image for each image individually, then this number was subtracted from every experimental image. Fig. 5 shows an example of the thus processed images for the wavelengths: $481 \mathrm{~nm}$, $505 \mathrm{~nm}, 550 \mathrm{~nm}$.

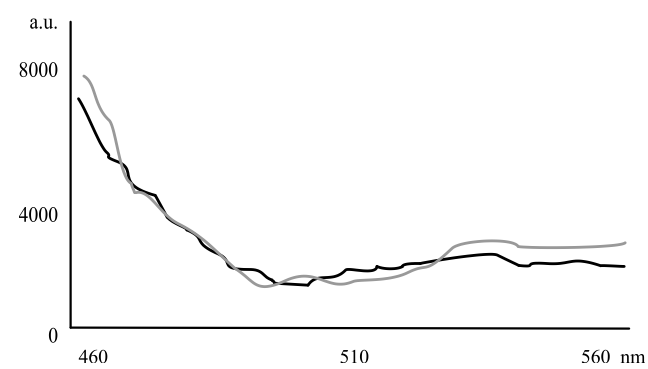

Fig. 7. - Diode spectrum obtained by means of spectrometer and by the results of processing a set of experimental images according to the simplified method

Based on the obtained images, a spectral distribution for each dot of the image can be obtained (fig. 7).

Fig. 7 shows a certain deterioration (in comparison with fig. 6) of the correspondence of a spectrum obtained on the spectrometer (dark line) and a spectrum obtained after processing the image sequence, formed by the diffraction lens. RMSE in this case was $13 \%$. Which is $2 \%$ more than RMSE of the exact solution. However,

Information Technology and Nanotechnology (ITNT-2015) 
the processing time here significantly reduces and in certain cases, it is possible to use a simplified algorithm to obtain spectral images of compact objects.

Spectra obtained for the different LED dots are a little bit different (fig. 8). Various dots at which the spectrum was measured were marked in fig. $8 \mathrm{a}$. The spectrum for a point marked with an arrow is shown in Fig. 7. The spectra for the points marked with a triangle and square, are shown in Fig. 6bc, respectively.

For fig. $8 \mathrm{~b}$ and $8 \mathrm{c}$ graphics differ slightly from the graphic in Fig. 7, and RMSE value is about $13 \%$.

a)
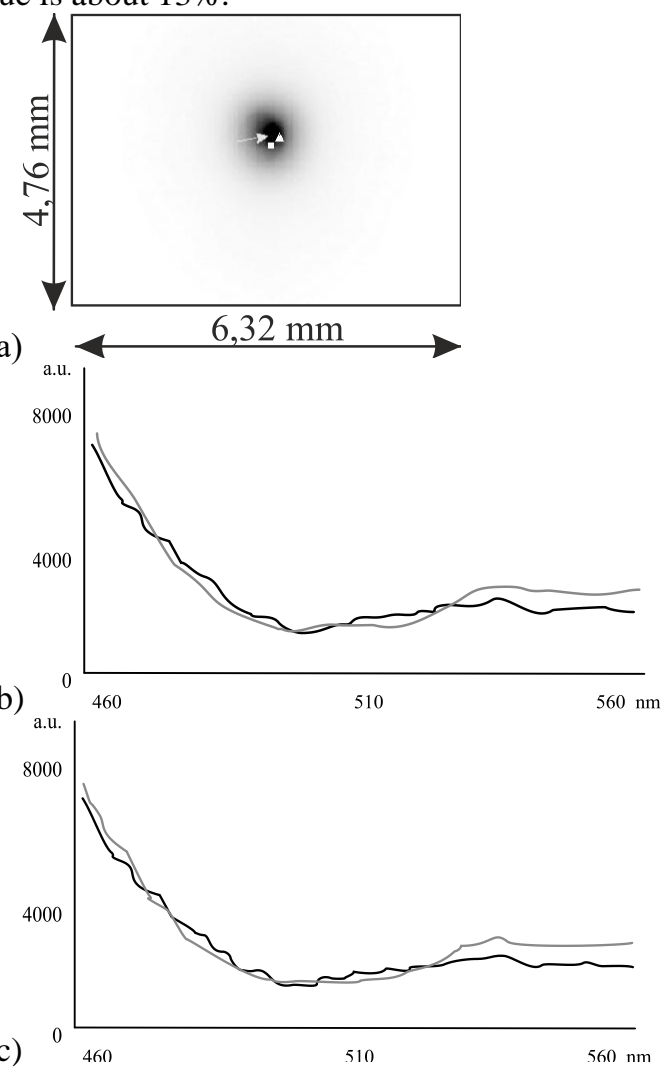

Fig. 8. - The diode spectrum obtained using a spectrometer and by the results of processing a set of experimental images by simplified method

Let us also consider the spectral image of the structure of the green, red and white LEDs in a cross shape, a white diode is situated in the center, green diodes are in vertical direction, and red diodes are in horizontal direction (Fig. 9). The spectra of these diodes are quite different from each other, and obtaining the spectral image of such object, we hereby confirm that the spectrometer based on diffractive lens is indeed imaging. 
a)

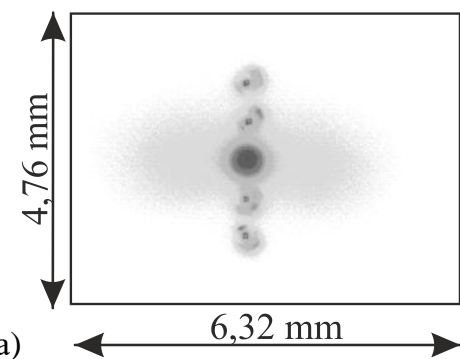

b)

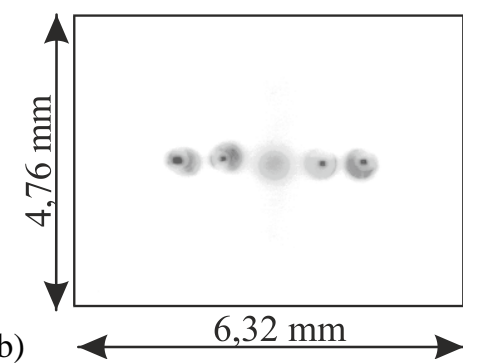

Fig. 9. - Image of patterns of green, red and white diode (in the center). (a) position of the matrix, wherein the green diodes are visible, (b) the position of the matrix, wherein the red diodes are visible

In Fig. 9, it is clearly seen that images of the red and green diodes are formed at different distances from the lens. Fig. 10 shows the spectra obtained for red and green diodes in comparison with their spectra measured on MS7501.

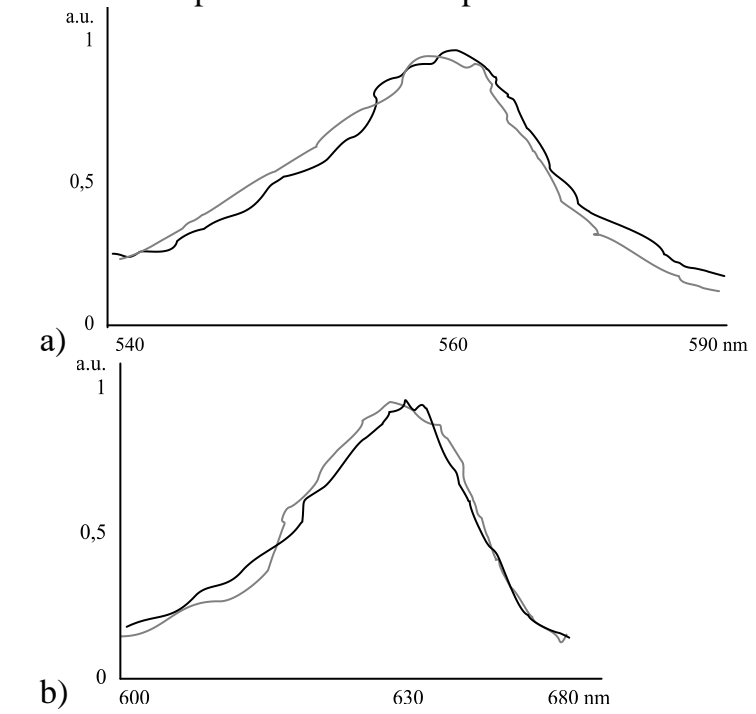

Fig. 10. - Spectrum of the green diode obtained in the experiment in comparison with its spectrum measured on MS7501 (a), the spectrum of the red diode obtained in the experiment in comparison with its spectrum measured on MS7501 (b)

The value of RMSE in fig. $10 \mathrm{a}-8 \%$, in fig. $10 \mathrm{~b}-11 \%$. The RMSE value obtained in the experiment is rather large $8-13 \%$, but we must consider three things:

1. spectrum of a single dot on the spectral image should not be identical with the spectrum of the entire diode (fig. 8b and 8c) (spectrometer measures the integral characteristics);

2. only a part of the spectrum was studied, but CCD array is also sensitive to components outside the measured range, therefore, they also introduce distortions 
in the spectrum reconstruction. In the future this can be avoided by installing the cutoff light filters;

3. the usual error level of imaging spectrometers is quite large compared to the errors of non-imaging spectrometers (1-2\%). As a rule, manufacturers of hyperspectral cameras indicate an error in the measurement of the spectrum in the range of 5$10 \%$ [17]. I.e. in our experiment, results that can be compared in accuracy are obtained with the obviously more simple design.

When working with more complex images in the future, a precise method based on system should be used (2).

However, based on the obtained results, we can talk about the working efficiency of this simple imaging spectrometer, consisting of a single diffractive lens.

\section{Conclusion}

The error in operation of the simplest imaging spectrometer, where the imaging system and the dispersion element are integrated, is investigated - spectrometer is based on diffractive lens.

The experimental results on the formation of the spectral image from the series of images formed by the diffraction lens, allow us to speak about the error of forming spectral images using diffractive lens with an error of $8-13 \%$, which roughly corresponds to the error of the existing samples of hyperspectral cameras.

\section{Funding Information}

The work was funded RSF grant 14-19-00114.

\section{Reference}

1. Voropay ES, Gulis IM, Kupreev AG. The dispersion hyperspectrometer with reconfigurable input aperture on the basis of the micro-mirror matrix. Herald BSU, 2009; 1(3): 31-35.

2. Kazanskiy NL, Kharitonov SI, Khonina SN, Volotovskiy SG, Strelkov YuS. Simulation of hyperspectrometer on spectral linear variable filters. Computer Optics, 2014; 38(2): 256-270.

3. Kazanskiy NL, Kharitonov SI, Karsakov AV, Khonina SN. Modeling action of a hyperspectrometer based on the Offner scheme within geometric optics. Computer Optics, 2014; 38 (2): 271-280.

4. Khonina SN, Ustinov AV, Skidanov RV. Binary lens: investigation of local focuses. Computer Optics, 2011; 35(3): 339-346.

5. Buralli DA, Morris GM. Design of diffractive singlets for monochromatic imaging. Applied optics, 1991; 30(16): 2151-2158.

6. Kazanskiy NL, Khonina SN, Skidanov RV, Morozov AA, Kharitonov SI, Volotovskiy SG. Formation of images using multilevel diffractive lens. Computer Optics, 2014; 38(3): 425-434.

7. Motogaito A, Hiramatsu K. Fabrication of Binary Diffractive Lenses and the Application to LED Lighting for Controlling Luminosity Distribution. Optical and Photonics Journal, 2013; 3(1): 67-73. 
8. Miyamoto K. The phase Fresnel lens. Journal of the Optical Society of America, 1961; 51(1): 17-20.

9. Faklis D, Morris GM. Spectral properties of multiorder diffractive lenses. Applied Optics, 1995; 34(14): 2462-2468.

10. Kitaura N, Ogata S, Mori Y. Spectrometer employing a micro-Fresnel lens. Optical Engineering, 1995; 34: 584-588.

11. Park Y. Miniaturization of a Fresnel spectrometer. Journal of Optics A: Pure and Applied Optics, 2008; 10: 095301.

12. Yang C, Edwards P, Shi K, Liu Z. Proposal and demonstration of a spectrometer using a diffractive optical element with dual dispersion and focusing functionality. Optical Letters, 2011; 36: 2023-2025.

13. Yang C, Shi K, Edwards P, Liu Z. Demonstration of a PDMS based hybrid grating and Fresnel lens (G-Fresnel) device. Optics Express, 2010; 18(23): 23529-23534.

14. Lyons D. Image spectrometry with a diffractive optic. Proceedings of SPIE, 1995; 2480: 123-131.

15. Lyons D, Whitcomb K. The DOE in "DOIS" a diffractive optic image spectrometer. Proceedings of SPIE, 1996; 2689: 274-283.

16. Petropavlovskiy U. The application features CCD interline transfer. Components and technologies, 2009; 5: 17-24.

17. Special Design Bureau of Instrument. Source: <http://www.kbsp.ru/ projects/detail.php? ELEMENT_ID=12>. 